\title{
Optimal Bounds for Neuman Mean Using Arithmetic and Centroidal Means
}

\author{
Ying-Qing Song, ${ }^{1}$ Wei-Mao Qian, ${ }^{2}$ and Yu-Ming $\mathrm{Chu}^{1}$ \\ ${ }^{1}$ School of Mathematics and Computation Sciences, Hunan City University, Yiyang 413000, China \\ ${ }^{2}$ School of Distance Education, Huzhou Broadcast and TV University, Huzhou 313000, China
}

Correspondence should be addressed to Yu-Ming Chu; chuyuming2005@126.com

Received 13 September 2015; Accepted 11 January 2016

Academic Editor: Henryk Hudzik

Copyright (C) 2016 Ying-Qing Song et al. This is an open access article distributed under the Creative Commons Attribution License, which permits unrestricted use, distribution, and reproduction in any medium, provided the original work is properly cited.

We present the best possible parameters $\alpha_{1}, \alpha_{2}, \beta_{1}, \beta_{2} \in \mathbb{R}$ and $\alpha_{3}, \beta_{3} \in(1 / 2,1)$ such that the double inequalities $\alpha_{1} A(a, b)+(1-$ $\left.\alpha_{1}\right) C(a, b)<N_{Q A}(a, b)<\beta_{1} A(a, b)+\left(1-\beta_{1}\right) C(a, b), A^{\alpha_{2}}(a, b) C^{1-\alpha_{2}}(a, b)<N_{Q A}(a, b)<A^{\beta_{2}}(a, b) C^{1-\beta_{2}}(a, b)$, and $C\left[\alpha_{3} a+(1-\right.$ $\left.\left.\alpha_{3}\right) b, \alpha_{3} b+\left(1-\alpha_{3}\right) a\right]<N_{\mathrm{QA}}(a, b)<C\left[\beta_{3} a+\left(1-\beta_{3}\right) b, \beta_{3} b+\left(1-\beta_{3}\right) a\right]$ hold for all $a, b>0$ with $a \neq b$ and give several sharp inequalities involving the hyperbolic and inverse hyperbolic functions. Here, $N(a, b), A(a, b), Q(a, b)$, and $C(a, b)$ are, respectively, the Neuman, arithmetic, quadratic, and centroidal means of $a$ and $b$, and $N_{\mathrm{QA}}(a, b)=N[Q(a, b), A(a, b)]$.

\section{Introduction}

Let $a, b>0$ with $a \neq b$. Then, the Schwab-Borchardt mean $\operatorname{SB}(a, b)[1-7]$ of $a$ and $b$ is given by

$$
\operatorname{SB}(a, b)= \begin{cases}\frac{\sqrt{b^{2}-a^{2}}}{\arccos (a / b)}, & a<b, \\ \frac{\sqrt{a^{2}-b^{2}}}{\cosh ^{-1}(a / b)}, & a>b,\end{cases}
$$

where $\cosh ^{-1}(x)=\log \left(x+\sqrt{x^{2}-1}\right)$ is the inverse hyperbolic cosine functions.

It is well known that the Schwab-Borchardt mean $\operatorname{SB}(a, b)$ is strictly increasing in both $a$ and $b$, nonsymmetric, and homogeneous of degree 1 with respect to $a$ and $b$. Many symmetric bivariate means are special cases of the SchwabBorchardt mean. For example, $P(a, b)=(a-b) /[2 \arcsin ((a-$ $b) /(a+b))]=\operatorname{SB}[G(a, b), A(a, b)]$ is the first Seiffert mean, $T(a, b)=(a-b) /[2 \arctan ((a-b) /(a+b))]=$ $\mathrm{SB}[A(a, b), \mathrm{Q}(a, b)]$ is the second Seiffert mean, $M(a, b)=$ $(a-b) /\left[2 \sinh ^{-1}((a-b) /(a+b))\right]=\operatorname{SB}[Q(a, b), A(a, b)]$ is the Neuman-Sándor mean, and $L(a, b)=(a-b) /\left[2 \tanh ^{-1}((a-\right.$ $b) /(a+b))]=\operatorname{SB}[A(a, b), G(a, b)]$ is the logarithmic mean, where $\sinh ^{-1}(x)=\log \left(x+\sqrt{1+x^{2}}\right)$ is the inverse hyperbolic sine function, $\tanh ^{-1}(x)=\log ((1+x) /(1-x)) / 2$ is the inverse hyperbolic tangent function, $G(a, b)=\sqrt{a b}, A(a, b)=$ $(a+b) / 2$, and $Q(a, b)=\sqrt{\left(a^{2}+b^{2}\right) / 2}$ are, respectively, the geometric, arithmetic, and quadratic means of $a$ and $b$, and the inequalities

$$
\begin{gathered}
H(a, b)<G(a, b)<L(a, b)<P(a, b)<A(a, b) \\
<M(a, b)<T(a, b)<Q(a, b)
\end{gathered}
$$

hold for all $a, b>0$ with $a \neq b$ (see [1]), where $H(a, b)=$ $2 a b /(a+b)$ is the harmonic mean of $a$ and $b$.

Recently, the Schwab-Borchardt mean and its generated means have been the subject intensive research. In particular, many remarkable inequalities for these means can be found in the literature $[1-5,8-16]$.

Liu and Meng [17] proved that the double inequality

$$
\begin{gathered}
\alpha C(a, b)+(1-\alpha) H(a, b)<T(a, b) \\
<\beta C(a, b)+(1-\beta) H(a, b)
\end{gathered}
$$

holds for all $a, b>0$ with $a \neq b$ if and only if $\alpha \leq 3 / \pi$ and $\beta \geq 1$, where

$$
C(a, b)=\frac{2\left(a^{2}+a b+b^{2}\right)}{3(a+b)}
$$

is the centroidal mean of $a$ and $b$. 
Very recently, Neuman [18] introduced the Neuman mean

$$
N(a, b)=\frac{1}{2}\left[a+\frac{b^{2}}{\mathrm{SB}(a, b)}\right],
$$

presented the explicit formula for $N_{\mathrm{QA}}(a, b)$ as

$$
\begin{aligned}
N_{\mathrm{QA}}(a, b) & \triangleq N[Q(a, b), A(a, b)] \\
& =\frac{1}{2} A(a, b)\left[\sqrt{1+v^{2}}+\frac{\sinh ^{-1}(v)}{v}\right],
\end{aligned}
$$

and proved that the double inequality

$$
M(a, b)<N_{\mathrm{QA}}(a, b)<T(a, b)
$$

holds for all $a, b>0$ with $a \neq b$, where $v=(a-b) /(a+b)$.

From (2), (3), and (7), we clearly see that the double inequality

$$
A(a, b)<N_{\mathrm{QA}}(a, b)<C(a, b)
$$

holds for all $a, b>0$ with $a \neq b$.

Let $J(x)=C[x a+(1-x) b, x b+(1-x) a]$. Then, it is not difficult to verify that the function $J(x)$ is continuous and strictly increasing on $[1 / 2,1]$ for fixed $a, b>0$ with $a \neq b$. Note that

$$
J\left(\frac{1}{2}\right)=A(a, b)<N_{\mathrm{QA}}(a, b)<C(a, b)=J(1)
$$

for all $a, b>0$ with $a \neq b$.

Motivated by inequalities (8) and (9), it is natural to ask what are best possible parameters $\alpha_{1}, \alpha_{2}, \beta_{1}, \beta_{2} \in \mathbb{R}$ and $\alpha_{3}, \beta_{3} \in(1 / 2,1)$ such that the double inequalities

$$
\begin{aligned}
& \alpha_{1} A(a, b)+\left(1-\alpha_{1}\right) C(a, b)<N_{\mathrm{QA}}(a, b) \\
& \quad<\beta_{1} A(a, b)+\left(1-\beta_{1}\right) C(a, b), \\
& A^{\alpha_{2}}(a, b) C^{1-\alpha_{2}}(a, b)<N_{\mathrm{QA}}(a, b) \\
& \quad<A^{\beta_{2}}(a, b) C^{1-\beta_{2}}(a, b), \\
& C\left[\alpha_{3} a+\left(1-\alpha_{3}\right) b, \alpha_{3} b+\left(1-\alpha_{3}\right) a\right]<N_{\mathrm{QA}}(a, b) \\
& \quad<C\left[\beta_{3} a+\left(1-\beta_{3}\right) b, \beta_{3} b+\left(1-\beta_{3}\right) a\right]
\end{aligned}
$$

hold for all $a, b>0$ with $a \neq b$ ? The main purpose of this paper is to answer these questions.

\section{Lemmas}

In order to prove our main results, we need several lemmas, which we present in this section.

Lemma 1 (see [19-21]). For $a, b \in \mathbb{R}$, let $f, g:[a, b] \rightarrow \mathbb{R}$ be continuous on $[a, b]$ and be differentiable on $(a, b)$, and let $g^{\prime}(x) \neq 0$ on $(a, b)$. If $f^{\prime}(x) / g^{\prime}(x)$ is increasing (decreasing) on $(a, b)$, then so are

$$
\begin{aligned}
& \frac{f(x)-f(a)}{g(x)-g(a)}, \\
& \frac{f(x)-f(b)}{g(x)-g(b)} .
\end{aligned}
$$

If $f^{\prime}(x) / g^{\prime}(x)$ is strictly monotone, then the monotonicity in the conclusion is also strict.

Lemma 2 (see [22-24]). Let $f(x)=\sum_{n=0}^{\infty} a_{n} x^{n}$ and $g(x)=$ $\sum_{n=0}^{\infty} b_{n} x^{n}$ be two real power series converging on $\mathbb{R}$ with $b_{n}>0$ for all $n \in \mathbb{N}$. Then, the following statements are true:

(1) If the nonconstant sequence $\left\{a_{n} / b_{n}\right\}$ is increasing (decreasing) for all $n \in \mathbb{N}$, then the function $x \mapsto$ $f(x) / g(x)$ is strictly increasing (decreasing) on $(0, \infty)$.

(2) If there exists $m \geq 1$ such that the nonconstant sequence $\left\{a_{n} / b_{n}\right\}$ is increasing (decreasing) for $0 \leq n \leq m$ and decreasing (increasing) for $n \geq m$, then there exists $x_{0} \in(0, \infty)$ such that the function $x \mapsto f(x) / g(x)$ is strictly increasing (decreasing) on $\left(0, x_{0}\right)$ and strictly decreasing (increasing) on $\left(x_{0}, \infty\right)$.

Lemma 3. The function

$$
\begin{aligned}
& f(x) \\
& =\frac{6 x+6 x \cosh (2 x)+(1 / 2) \sinh (4 x)-7 \sinh (2 x)}{-4 x+4 x \cosh (2 x)+\sinh (4 x)-2 \sinh (2 x)}
\end{aligned}
$$

is strictly increasing from $(0, \log (1+\sqrt{2}))$ onto $(1 / 2,[3 \log (1+$ $\sqrt{2})-\sqrt{2}] /[\log (1+\sqrt{2})+\sqrt{2}])$.

Proof. Making use of the power series formulas $\sinh (x)=$ $\sum_{n=0}^{\infty} x^{2 n+1} /(2 n+1)$ ! and $\cosh (x)=\sum_{n=0}^{\infty} x^{2 n} /(2 n)$ !, we have

$$
\begin{aligned}
f(x) & =\frac{6 x+6 x \sum_{n=0}^{\infty}\left(2^{2 n} /(2 n) !\right) x^{2 n}+(1 / 2) \sum_{n=0}^{\infty}\left(4^{2 n+1} /(2 n+1) !\right) x^{2 n+1}-7 \sum_{n=0}^{\infty}\left(2^{2 n+1} /(2 n+1) !\right) x^{2 n+1}}{-4 x+4 x \sum_{n=0}^{\infty}\left(2^{2 n} /(2 n) !\right) x^{2 n}+\sum_{n=0}^{\infty}\left(4^{2 n+1} /(2 n+1) !\right) x^{2 n+1}-2 \sum_{n=0}^{\infty}\left(2^{2 n+1} /(2 n+1) !\right) x^{2 n+1}} \\
& =\frac{\sum_{n=0}^{\infty}\left(2^{2 n+4}\left(2^{2 n+1}+3 n+1\right) /(2 n+3) !\right) x^{2 n}}{\sum_{n=0}^{\infty}\left(2^{2 n+5}\left(2^{2 n+1}+n+1\right) /(2 n+3) !\right) x^{2 n}} .
\end{aligned}
$$


Let

$$
\begin{aligned}
& c_{n}=\sum_{n=0}^{\infty} \frac{2^{2 n+4}\left(2^{2 n+1}+3 n+1\right)}{(2 n+3) !}, \\
& d_{n}=\sum_{n=0}^{\infty} \frac{2^{2 n+5}\left(2^{2 n+1}+n+1\right)}{(2 n+3) !} .
\end{aligned}
$$

Then, we clearly see that

$$
d_{n}>0
$$

for all $n \geq 0$ and

$$
\frac{c_{n+1}}{d_{n+1}}-\frac{c_{n}}{d_{n}}=\frac{1+(1-3 n) 2^{2 n+1}}{\left(2^{2 n+1}+n+1\right)\left(2^{2 n+3}+n+2\right)} .
$$

Let

$$
e_{n}=1+(1-3 n) 2^{2 n+1} \text {. }
$$

Then, simple computations lead to

$$
\begin{aligned}
& e_{0}=3>0, \\
& e_{n}<1-2^{2 n+2}<0
\end{aligned}
$$

for all $n \geq 1$.

It follows from (16)-(18) that the nonconstant sequence $\left\{c_{n} / d_{n}\right\}$ is increasing for $0 \leq n \leq 1$ and decreasing for $n \geq 1$. Then, from (13)-(15) and Lemma 2(2) we know that there exists $x_{0} \in(0, \infty)$ such that $f(x)$ is strictly increasing on $\left(0, x_{0}\right)$ and strictly decreasing on $\left(x_{0}, \infty\right)$.

Differentiating $f(x)$ gives

$$
\begin{aligned}
f^{\prime}(x)= & \frac{6-8 \cosh (2 x)+12 x \sinh (2 x)+2 \cosh (4 x)}{-4 x+4 x \cosh (2 x)+\sinh (4 x)-2 \sinh (2 x)} \\
& -\frac{[6 x+6 x \cosh (2 x)+(1 / 2) \sinh (4 x)-7 \sinh (2 x)][-4+8 x \sinh (2 x)+4 \cosh (4 x)]}{[-4 x+4 x \cosh (2 x)+\sinh (4 x)-2 \sinh (2 x)]^{2}}, \\
f^{\prime}[\log (1+\sqrt{2})]= & \frac{6-24+24 \sqrt{2} \log (1+\sqrt{2})+34}{-4 \log (1+\sqrt{2})+12 \log (1+\sqrt{2})+12 \sqrt{2}-4 \sqrt{2}} \\
& -\frac{[6 \log (1+\sqrt{2})+18 \log (1+\sqrt{2})+6 \sqrt{2}-14 \sqrt{2}][-4+16 \sqrt{2} \log (1+\sqrt{2})+68]}{[-4 \log (1+\sqrt{2})+12 \log (1+\sqrt{2})+12 \sqrt{2}-4 \sqrt{2}]^{2}} \\
= & \frac{10 \sqrt{2}-12 \log (1+\sqrt{2})-3 \sqrt{2} \log ^{2}(1+\sqrt{2})}{[\sqrt{2}+\log (1+\sqrt{2})]^{2}} .
\end{aligned}
$$

Note that

$$
\begin{aligned}
& 10 \sqrt{2}-12 \log (1+\sqrt{2})-3 \sqrt{2} \log ^{2}(1+\sqrt{2}) \\
& \quad=0.2698 \cdots>0, \\
& f\left(0^{+}\right)=\frac{c_{0}}{d_{0}}=\frac{1}{2}, \\
& f[\log (1+\sqrt{2})]=\frac{3 \log (1+\sqrt{2})-\sqrt{2}}{\log (1+\sqrt{2})+\sqrt{2}} .
\end{aligned}
$$

It follows from (19) and (20) together with the piecewise monotonicity of $f(x)$ that $\log (1+\sqrt{2})<x_{0}$ and $f(x)$ is strictly increasing on $(0, \log (1+\sqrt{2}))$.
Therefore, Lemma 3 follows from (21) and the monotonicity of $f(x)$ on the interval $(0, \log (1+\sqrt{2}))$.

Lemma 4. The function

$$
g(x)=\frac{\sinh (x)[2-\cosh (x)]-x}{\sinh ^{3}(x)}
$$

is strictly increasing from $(0, \log (1+\sqrt{2}))$ onto $(-1 / 3,2-\sqrt{2}-$ $\log (1+\sqrt{2}))$.

Proof. Making use of the power series formulas $\sinh (x)=$ $\sum_{n=0}^{\infty} x^{2 n+1} /(2 n+1)$ ! and $\cosh (x)=\sum_{n=0}^{\infty} x^{2 n} /(2 n+)$ ! together with the identity $4 \sinh ^{3}(x)=\sinh (3 x)-3 \sinh (x)$, one has

$$
\begin{aligned}
g(x) & =\frac{2 \sinh (x)-(1 / 2) \sinh (2 x)-x}{(1 / 4)[\sinh (3 x)-3 \sinh (x)]}=\frac{2 \sum_{n=0}^{\infty}(1 /(2 n+1) !) x^{2 n+1}-(1 / 2) \sum_{n=0}^{\infty}\left(2^{2 n+1} /(2 n+1) !\right) x^{2 n+1}-x}{(1 / 4)\left[\sum_{n=0}^{\infty}\left(3^{2 n+1} /(2 n+1) !\right) x^{2 n+1}-3 \sum_{n=0}^{\infty}(1 /(2 n+1) !) x^{2 n+1}\right]} \\
& =\frac{\sum_{n=0}^{\infty}\left(\left(2-2^{2 n+2}\right) /(2 n+3) !\right) x^{2 n}}{\sum_{n=0}^{\infty}\left(\left(3^{2 n+3}-3\right) /(4 \times(2 n+3) !)\right) x^{2 n}} .
\end{aligned}
$$


Let

$$
\begin{aligned}
& u_{n}=\frac{2-2^{2 n+2}}{(2 n+3) !}, \\
& v_{n}=\frac{3^{2 n+3}-3}{4 \times(2 n+3) !} .
\end{aligned}
$$

Then,

$$
\begin{aligned}
& \frac{u_{n+1}}{v_{n+1}}-\frac{u_{n}}{v_{n}}=\frac{8\left[\left(10 \times 2^{2 n}-8\right) 3^{2 n+1}+2^{2 n+1}\right]}{\left(3^{2 n+4}-1\right)\left(3^{2 n+2}-1\right)}>0,, \\
& v_{n}>0,
\end{aligned}
$$

for all $n \geq 0$. Note that

$$
\begin{gathered}
g\left(0^{+}\right)=\frac{u_{0}}{v_{0}}=-\frac{1}{3}, \\
g[\log (1+\sqrt{2})]=2-\sqrt{2}-\log (1+\sqrt{2}) .
\end{gathered}
$$

Therefore, Lemma 4 follows easily from (23)-(26) and Lemma 2(1).

\section{Main Results}

Theorem 5. The double inequality

$$
\begin{gathered}
\alpha_{1} A(a, b)+\left(1-\alpha_{1}\right) C(a, b)<N_{\mathrm{QA}}(a, b) \\
<\beta_{1} A(a, b)+\left(1-\beta_{1}\right) C(a, b)
\end{gathered}
$$

holds for all $a, b>0$ with $a \neq b$ if and only if $\alpha_{1} \geq 4-3[\sqrt{2}+$ $\log (1+\sqrt{2})] / 2=0.5566 \cdots$ and $\beta_{1} \leq 1 / 2$.

Proof. Since all the means $A(a, b), N_{\mathrm{QA}}(a, b)$, and $C(a, b)$ are symmetric and homogeneous of degree 1 , without loss of generality, we assume that $a>b$. Let $v=(a-b) /(a+b) \epsilon$ $(0,1)$. Then, (4) leads to

$$
C(a, b)=\left(1+\frac{1}{3} v^{2}\right) A(a, b) .
$$

From (6) and (28), we clearly see that inequality (27) is equivalent to

$$
\beta_{1}<\frac{2 v^{2}-3\left(\sqrt{1+v^{2}}+\left(\sinh ^{-1}(v)\right) / v\right)+6}{2 v^{2}}<\alpha_{1} .
$$

Let $x=\sinh ^{-1}(v)$. Then, $x \in(0, \log (1+\sqrt{2}))$ and

$$
\begin{gathered}
\frac{2 v^{2}-3\left(\sqrt{1+v^{2}}+\left(\sinh ^{-1}(v)\right) / v\right)+6}{2 v^{2}} \\
=\frac{\sinh (3 x)-3 \sinh (2 x)+9 \sinh (x)-6 x}{\sinh (3 x)-3 \sinh (x)} \\
=1+\frac{3[\sinh (x)(2-\cosh (x))-x]}{2 \sinh ^{3}(x)} .
\end{gathered}
$$

Therefore, Theorem 5 follows easily from (29) and (30) together with Lemma 4.

Theorem 6. The double inequality

$$
\begin{aligned}
A^{\alpha_{2}}(a, b) C^{1-\alpha_{2}}(a, b) & <N_{\mathrm{QA}}(a, b) \\
& <A^{\beta_{2}}(a, b) C^{1-\beta_{2}}(a, b)
\end{aligned}
$$

holds for all $a, b>0$ with $a \neq b$ if and only if $\alpha_{2} \geq 1-[\log (\sqrt{2}+$ $\log (1+\sqrt{2}))-\log 2] /[2 \log 2-\log 3]=0.5208 \cdots$ and $\beta_{2} \leq$ $1 / 2$.

Proof. Without loss of generality, we assume that $a>b$. Let $v=(a-b) /(a+b) \in(0,1)$. Then, from (6) and (28) we clearly see that inequality (31) is equivalent to

$\beta_{2}$

$$
\begin{aligned}
& <\frac{\log \left(v^{2}+3\right)-\log \left[\sqrt{1+v^{2}}+\left(\sinh ^{-1}(v)\right) / v\right]-\log 3+\log 2}{\log \left(v^{2}+3\right)-\log 3} \\
& <\alpha_{2} .
\end{aligned}
$$

$$
\text { Let } x=\sinh ^{-1}(v) \text {. Then, } x \in(0, \log (1+\sqrt{2})) \text {, and }
$$

$$
\begin{aligned}
& \frac{\log \left(v^{2}+3\right)-\log \left[\sqrt{1+v^{2}}+\left(\sinh ^{-1}(v)\right) / v\right]-\log 3+\log 2}{\log \left(v^{2}+3\right)-\log 3} \\
& \quad=\frac{\log \left[3+\sinh ^{2}(x)\right]-\log [x+\sinh (x) \cosh (x)]+\log [\sinh (x)]-\log 3+\log 2}{\log \left[3+\sinh ^{2}(x)\right]-\log 3}
\end{aligned}
$$

Let

$$
G(x)=\frac{\log \left[3+\sinh ^{2}(x)\right]-\log [x+\sinh (x) \cosh (x)]+\log [\sinh (x)]-\log 3+\log 2}{\log \left[3+\sinh ^{2}(x)\right]-\log 3}
$$




$$
\begin{aligned}
& g_{1}(x)=\log \left[3+\sinh ^{2}(x)\right]-\log [x+\sinh (x) \cosh (x)]+\log [\sinh (x)]-\log 3+\log 2, \\
& g_{2}(x)=\log \left[3+\sinh ^{2}(x)\right]-\log 3 .
\end{aligned}
$$

Then, simple computations lead to

$$
\begin{aligned}
& g_{1}\left(0^{+}\right)=g_{2}\left(0^{+}\right)=0, \\
& \frac{g_{1}^{\prime}(x)}{g_{2}^{\prime}(x)} \\
& =\frac{6 x+6 x \cosh (2 x)+(1 / 2) \sinh (4 x)-7 \sinh (2 x)}{-4 x+4 x \cosh (2 x)+\sinh (4 x)-2 \sinh (2 x)} .
\end{aligned}
$$

It follows from (34)-(37) and Lemmas 1 and 3 that the function

$$
G(x)=\frac{g_{1}(x)}{g_{2}(x)}=\frac{g_{1}(x)-g_{1}\left(0^{+}\right)}{g_{2}(x)-g_{2}\left(0^{+}\right)}
$$

is strictly increasing on the interval $(0, \log (1+\sqrt{2}))$. Note that

$$
\begin{aligned}
& G\left(0^{+}\right)=\frac{g_{1}^{\prime}\left(0^{+}\right)}{g_{2}^{\prime}\left(0^{+}\right)}=\frac{c_{0}}{d_{0}}=\frac{1}{2}, \\
& G[\log (1+\sqrt{2})] \\
& \quad=1-\frac{\log [\sqrt{2}+\log (1+\sqrt{2})]-\log 2}{2 \log 2-\log 3},
\end{aligned}
$$

where $c_{n}$ and $d_{n}$ are defined by (14).

Therefore, Theorem 6 follows easily from (32)-(34) and (39) together with the monotonicity of $G(x)$ on the interval $(0, \log (1+\sqrt{2}))$.

Theorem 7. Let $\alpha_{3}, \beta_{3} \in(1 / 2,1)$. Then, the double inequality

$$
\begin{gathered}
C\left[\alpha_{3} a+\left(1-\alpha_{3}\right) b, \alpha_{3} b+\left(1-\alpha_{3}\right) a\right]<N_{\mathrm{QA}}(a, b) \\
<C\left[\beta_{3} a+\left(1-\beta_{3}\right) b, \beta_{3} b+\left(1-\beta_{3}\right) a\right]
\end{gathered}
$$

holds for all $a, b>0$ with $a \neq b$ if and only if $\alpha_{3} \leq 1 / 2+$ $\sqrt{6[\sqrt{2}+\log (1+\sqrt{2})]-12} / 4=0.8329 \cdots$ and $\beta_{3} \geq 1 / 2+$ $\sqrt{2} / 4=0.8535 \cdots$.

Proof. Without loss of generality, we assume that $a>b$. Let $v=(a-b) /(a+b) \in(0,1)$ and $p \in(1 / 2,1)$. Then, (4) and (6) lead to

$$
\begin{aligned}
C & {[p a+(1-p) b, p b+(1-p) a]-N_{\mathrm{QA}}(a, b)=\frac{1}{3} } \\
\cdot & A(a, b)\left[3+(2 p-1)^{2} v^{2}\right]-\frac{1}{2} A(a, b) \\
\cdot & {\left[\sqrt{1+v^{2}}+\left(\sinh ^{-1}(v)\right) / v\right]=A(a, b) } \\
\cdot & {\left[\frac{v\left(2-\sqrt{1+v^{2}}\right)-\sinh ^{-1}(v)}{2 v^{3}}+\frac{1}{3}(2 p-1)^{2}\right] v^{2} . }
\end{aligned}
$$

Let $x=\sinh ^{-1}(v)$. Then, $x \in(0, \log (1+\sqrt{2}))$ and

$$
\begin{aligned}
& \frac{v\left(2-\sqrt{1+v^{2}}\right)-\sinh ^{-1}(v)}{2 v^{3}} \\
& \quad=\frac{\sinh (x)[2-\cosh (x)]-x}{2 \sinh ^{3}(x)}=\frac{1}{2} g(x),
\end{aligned}
$$

where $g(x)$ is defined by Lemma 4 .

Therefore, Theorem 7 follows easily from (41) and (42) together with Lemma 4.

Let $a>b>0$ and $t=(\log a-\log b) / 2>0$. Then, we clearly see that

$$
\begin{aligned}
\frac{a-b}{a+b} & =\tanh (t), \\
\sqrt{1+\left(\frac{a-b}{a+b}\right)^{2}} & =\frac{\cosh ^{1 / 2}(2 t)}{\cosh (t)},
\end{aligned}
$$

and (4) and (6) lead to

$$
\begin{aligned}
& C(a, b)=\frac{4 \cosh ^{2}(t)-1}{3 \cosh ^{2}(t)} A(a, b), \\
& C[\alpha a+(1-\alpha) b, \alpha b+(1-\alpha) a] \\
& \quad=\frac{2\left(\alpha^{2}-\alpha+1\right) \cosh (2 t)+\left(1+2 \alpha-2 \alpha^{2}\right)}{3 \cosh ^{2}(t)} \\
& \cdot A(a, b), \\
& N_{\mathrm{QA}}(a, b)=\frac{1}{2}\left[\frac{\cosh ^{1 / 2}(2 t)}{\cosh ^{2}(t)}+\frac{\sinh ^{-1}(\tanh (t))}{\tanh (t)}\right] \\
& \cdot A(a, b) .
\end{aligned}
$$

From (44) and Theorems 5-7, we get Corollary 8 immediately.

Corollary 8. Let $\alpha_{1}, \alpha_{2}, \beta_{1}, \beta_{2} \in \mathbb{R}$ and $\alpha_{3}, \beta_{3} \in(1 / 2,1)$. Then, the double inequalities

$$
\begin{aligned}
& \frac{8-2 \alpha_{1}}{3}-\frac{2\left(1-\alpha_{1}\right)}{3 \cosh ^{2}(t)} \\
& <\frac{\cosh ^{1 / 2}(2 t)}{\cosh (t)}+\frac{\sinh ^{-1}(\tanh (t))}{\tanh (t)} \\
& <\frac{8-2 \beta_{1}}{3}-\frac{2\left(1-\beta_{1}\right)}{3 \cosh ^{2}(t)},
\end{aligned}
$$




$$
\begin{aligned}
& 2\left(\frac{4}{3}-\frac{1}{3 \cosh ^{2}(t)}\right)^{1-\alpha_{2}} \\
& <\frac{\cosh ^{1 / 2}(2 t)}{\cosh (t)}+\frac{\sinh ^{-1}(\tanh (t))}{\tanh (t)} \\
& <2\left(\frac{4}{3}-\frac{1}{3 \cosh ^{2}(t)}\right)^{1-\beta_{2}} \\
& \frac{4\left(\alpha_{3}^{2}-\alpha_{3}+1\right) \cosh (2 t)+2\left(1+2 \alpha_{3}-2 \alpha_{3}^{2}\right)}{3 \cosh ^{2}(t)} \\
& <\frac{\cosh ^{1 / 2}(2 t)}{\cosh (t)}+\frac{\sinh ^{-1}(\tanh (t))}{\tanh (t)} \\
& <\frac{4\left(\beta_{3}^{2}-\beta_{3}+1\right) \cosh (2 t)+2\left(1+2 \beta_{3}-2 \beta_{3}^{2}\right)}{3 \cosh ^{2}(t)}
\end{aligned}
$$

hold for all $t>0$ if and only if $\alpha_{1} \geq 4-3[\sqrt{2}+\log (1+\sqrt{2})] / 2$, $\beta_{1} \leq 1 / 2, \alpha_{2} \geq 1-[\log (\sqrt{2}+\log (1+\sqrt{2}))-\log 2] /[2 \log 2-$ $\log 3], \beta_{2} \leq 1 / 2, \alpha_{3} \leq 1 / 2+\sqrt{6[\sqrt{2}+\log (1+\sqrt{2})]-12} / 4$, and $\beta_{3} \geq 1 / 2+\sqrt{2} / 4$.

Let $x=\tanh (t) \in(0,1)$. Then, we clearly see that

$$
\begin{aligned}
\cosh (t) & =\frac{1}{\sqrt{1-x^{2}}}, \\
\cosh ^{1 / 2}(2 t) & =\sqrt{\frac{1+x^{2}}{1-x^{2}}},
\end{aligned}
$$

Corollary 9. Let $\alpha_{1}, \alpha_{2}, \beta_{1}, \beta_{2} \in \mathbb{R}$ and $\alpha_{3}, \beta_{3} \in(1 / 2,1)$. Then, the double inequalities

$$
\begin{aligned}
2 & +\frac{2\left(1-\alpha_{1}\right)}{3} x^{2}-\sqrt{1+x^{2}}<\frac{\sinh ^{-1}(x)}{x}<2 \\
& +\frac{2\left(1-\beta_{1}\right)}{3} x^{2}-\sqrt{1+x^{2}} \\
2\left(1+\frac{x^{2}}{3}\right)^{1-\alpha_{2}}-\sqrt{1+x^{2}}<\frac{\sinh ^{-1}(x)}{x} & \\
& <2\left(1+\frac{x^{2}}{3}\right)^{1-\beta_{2}}-\sqrt{1+x^{2}}, \\
4\left(\alpha_{3}^{2}-\alpha_{3}+1\right) & 3\left(1+x^{2}\right) \\
& +\frac{2\left(1+2 \alpha_{3}-2 \alpha_{3}^{2}\right)}{3}\left(1-x^{2}\right)-\sqrt{1+x^{2}} \\
& <\frac{\sinh ^{-1}(x)}{x}<\frac{4\left(\beta_{3}^{2}-\beta_{3}+1\right)}{3}\left(1+x^{2}\right) \\
& +\frac{2\left(1+2 \beta_{3}-2 \beta_{3}^{2}\right)}{3}\left(1-x^{2}\right)-\sqrt{1+x^{2}}
\end{aligned}
$$

hold for all $x \in(0,1)$ if and only if $\alpha_{1} \geq 4-3[\sqrt{2}+$ $\log (1+\sqrt{2})] / 2, \beta_{1} \leq 1 / 2, \alpha_{2} \geq 1-[\log (\sqrt{2}+\log (1+$ $\sqrt{2}))-\log 2] /[2 \log 2-\log 3], \beta_{2} \leq 1 / 2, \alpha_{3} \leq 1 / 2+$ $\sqrt{6[\sqrt{2}+\log (1+\sqrt{2})]-12} / 4$, and $\beta_{3} \geq 1 / 2+\sqrt{2} / 4$.

\section{Conflict of Interests}

The authors declare that there is no conflict of interests regarding the publication of this paper.

\section{Acknowledgments}

This research was supported by the Natural Science Foundation of China under Grants 11371125 and 61374086, the Natural Science Foundation of Zhejiang Province under Grant LY13A010004, the Natural Science Foundation of Hunan Province under Grant 12C0577, and the Natural Science Foundation of the Zhejiang Broadcast and TV University under Grant XKT-15G17.

\section{References}

[1] E. Neuman and J. Sándor, "On the Schwab-Borchardt mean," Mathematica Pannonica, vol. 14, no. 2, pp. 253-266, 2003.

[2] E. Neuman and J. Sándor, "On the Schwab-Borchardt mean II," Mathematica Pannonica, vol. 17, no. 1, pp. 49-59, 2006.

[3] E. Neuman, "Inequalities for the Schwab-Borchardt mean and their applications," Journal of Mathematical Inequalities, vol. 5, no. 4, pp. 601-609, 2011.

[4] E. Neuman, "On some means derived from the SchwabBorchardt mean," Journal of Mathematical Inequalities, vol. 8, no. 1, pp. 171-183, 2014.

[5] E. Neuman, "On some means derived from the SchwabBorchardt mean II," Journal of Mathematical Inequalities, vol. 8, no. 2, pp. 359-368, 2014.

[6] E. Neuman, "On the p-version of the Schwab-Borchardt mean," International Journal of Mathematics and Mathematical Sciences, vol. 2014, Article ID 697643, 7 pages, 2014.

[7] E. Neuman, "On the $p$-version of the Schwab-Borchardt mean II," International Journal of Mathematics and Mathematical Sciences, vol. 2015, Article ID 351742, 4 pages, 2015.

[8] E. Neuman, "A note on a certain bivariate mean," Journal of Mathematical Inequalities, vol. 6, no. 4, pp. 637-643, 2012.

[9] E. Neuman, "Sharp inequalities involving Neuman-Sándor and logarithmic means," Journal of Mathematical Inequalities, vol. 7, no. 3, pp. 413-419, 2013.

[10] E. Neuman, "Inequalities involving certain bivariate means II," Journal of Inequalities and Special Functions, vol. 4, no. 4, pp. 12-20, 2013.

[11] E. Neuman, "A one-parameter family of bivariate means," Journal of Mathematical Inequalities, vol. 7, no. 3, pp. 399-412, 2013.

[12] Y.-M. Chu and B.-Y. Long, "Bounds of the Neuman-Sándor mean using power and identric means," Abstract and Applied Analysis, vol. 2013, Article ID 832591, 6 pages, 2013.

[13] Z.-J. Guo, Y.-M. Chu, Y.-Q. Song, and X.-J. Tao, "Sharp bounds for Neuman means by harmonic, arithmetic, and contraharmonic means," Abstract and Applied Analysis, vol. 2014, Article ID 914242, 8 pages, 2014. 
[14] H. Sun, T.-H. Zhao, Y.-M. Chu, and B.-Y. Liu, "A note on the Neuman-Sándor mean," Journal of Mathematical Inequalities, vol. 8, no. 2, pp. 287-297, 2014.

[15] Y. Zhang, Y.-M. Chu, and Y.-L. Jiang, "Sharp geometric mean bounds for Neuman means," Abstract and Applied Analysis, vol. 2014, Article ID 949815, 6 pages, 2014.

[16] Y.-M. Chu and W.-M. Qian, "Refinements of bounds for Neuman means," Abstract and Applied Analysis, vol. 2014, Article ID 354132, 8 pages, 2014.

[17] H. Liu and X.-J. Meng, "The optimal convex combination bounds for the centroidal mean," in Integral Equations, Boundary Value Problems and Related Problems, pp. 258-262, World Science Publisher, Hackensack, NJ, USA, 2013.

[18] E. Neuman, “On a new bivariate mean," Aequationes Mathematicae, vol. 88, no. 3, pp. 277-289, 2014.

[19] H. Alzer and S.-L. Qiu, "Monotonicity theorems and inequalities for the complete elliptic integrals," Journal of Computational and Applied Mathematics, vol. 172, no. 2, pp. 289-312, 2004.

[20] G. D. Anderson, S.-L. Qiu, M. K. Vamanamurthy, and M. Vuorinen, "Generalized elliptic integrals and modular equation," Pacific Journal of Mathematics, vol. 192, no. 1, pp. 1-37, 2000.

[21] G. D. Anderson, M. K. Vamanamurthy, and M. K. Vuorinen, Conformal Invariants, Inequalities, and Quasiconformal Maps, John Wiley \& Sons, New York, NY, USA, 1997.

[22] F. Belzunce, E.-M. Ortega, and J. M. Ruiz, "On non-monotonic ageing properties from the Laplace transform, with actuarial applications," Insurance: Mathematics and Economics, vol. 40, no. 1, pp. 1-14, 2007.

[23] S. Simić and M. Vuorinen, "Landen inequalities for zerobalanced hypergeometric functions," Abstract and Applied Analysis, vol. 2012, Article ID 932061, 11 pages, 2012.

[24] Z.-H. Yang, Y.-M. Chu, and M.-K. Wang, "Monotonicity criterion for the quotient of power series with applications," Journal of Mathematical Analysis and Applications, vol. 428, no. 1, pp. 587-604, 2015. 


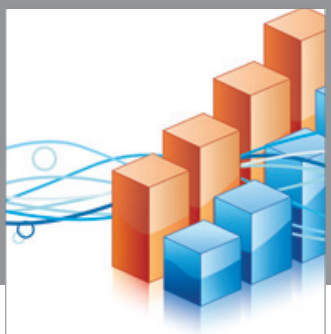

Advances in

Operations Research

vatem alat4

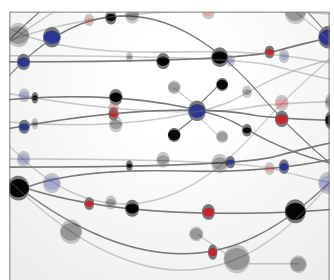

\section{The Scientific} World Journal
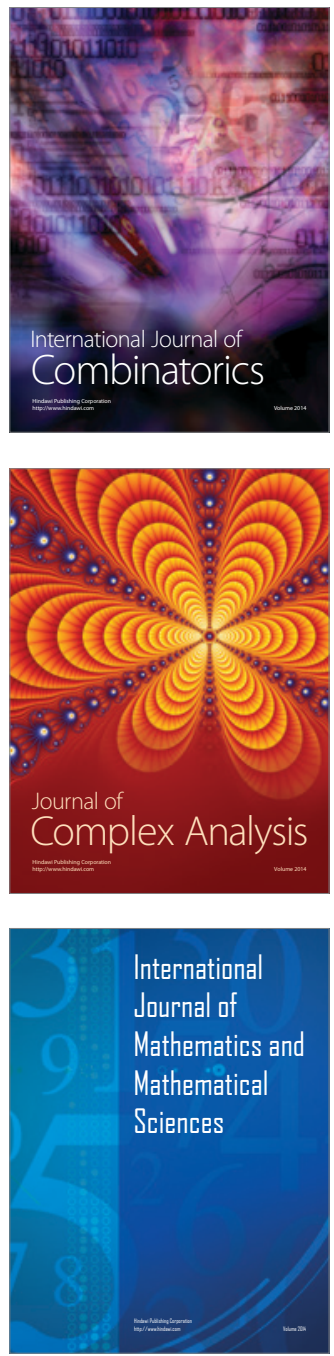
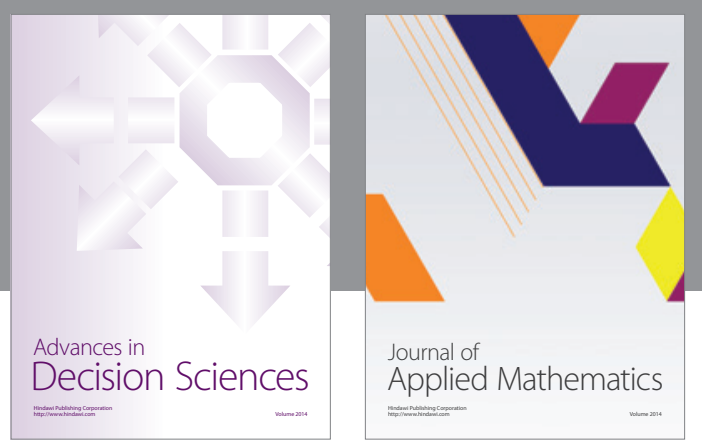

Algebra

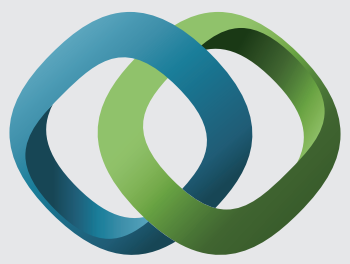

\section{Hindawi}

Submit your manuscripts at

http://www.hindawi.com
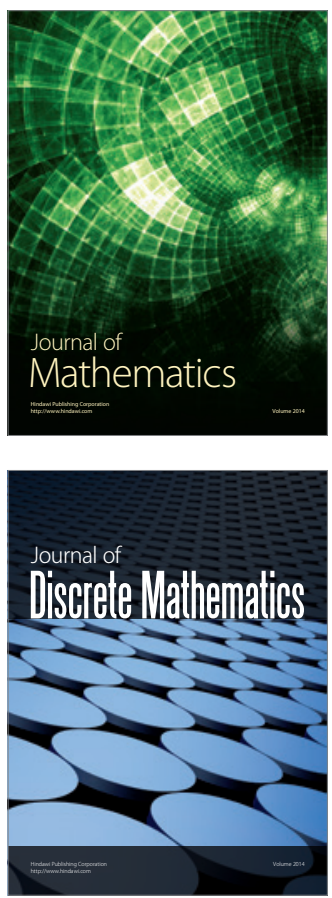

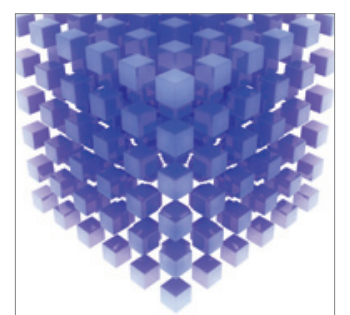

Mathematical Problems in Engineering
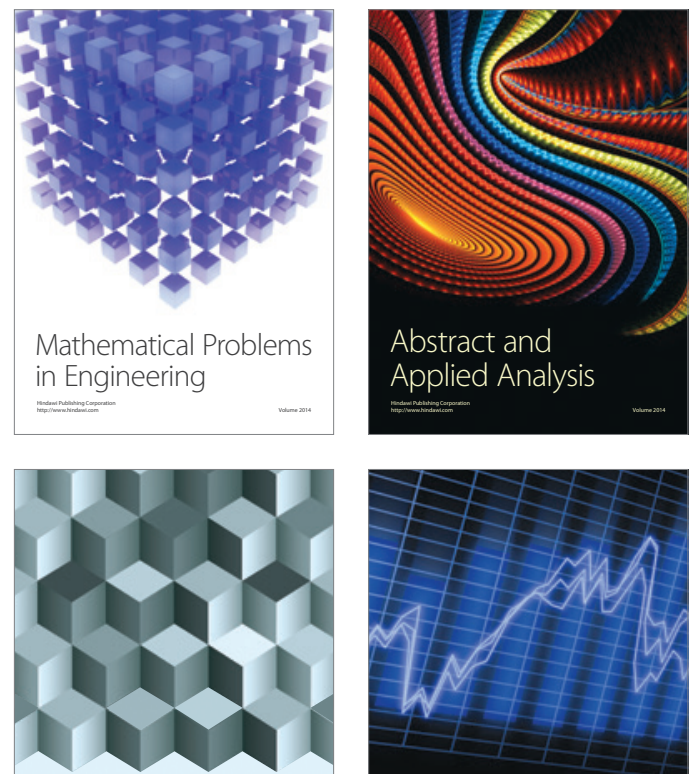

Journal of

Function Spaces

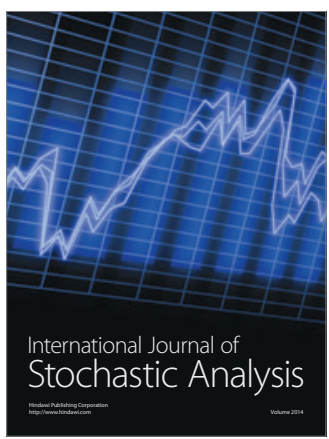

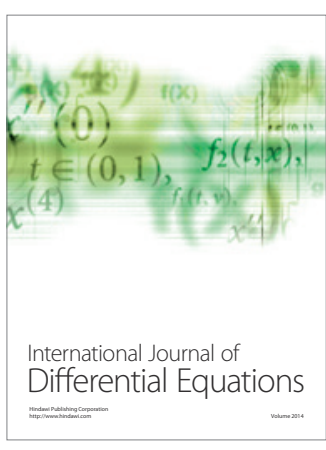
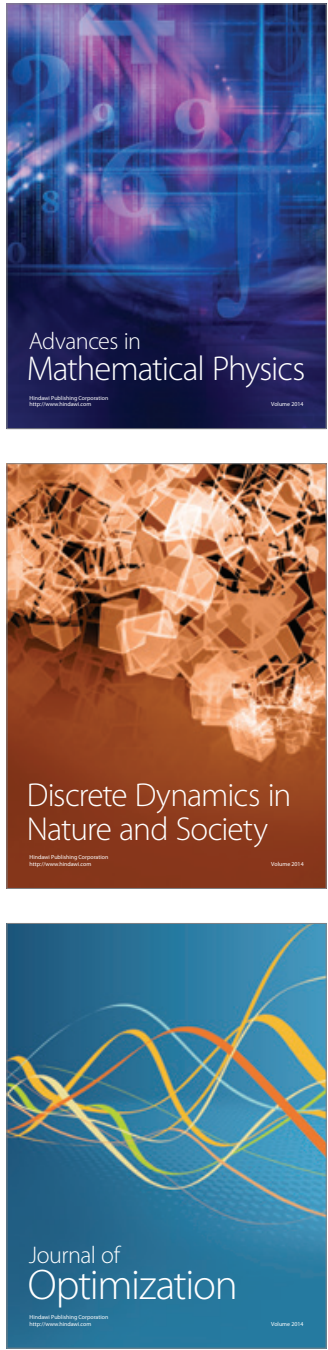